\title{
INTELLIGENT TRANSPORTATION SYSTEMS
}

Intelligent transportation system (ITS) stands for a transportation system with improved quality of control using an added "intelligence". Improved control can be attained by optimisation methods, by simualtions, interactive methods or artificial intelligence. Anyhow, a suitable model of the system, correct input data, optimisation routines and user-friendly environment for data input and output are typical parts of such a system. Basic parts of a transportation system, transportation processes and their models, optimisation methods and problems of implementation of an ITS are discussed in the paper in more detail.

\section{Introduction}

Intelligent transportation systems (further abbreviated as ITS) is a term used to describe transportation systems, which use a certain "intelligence" to improve the quality of their control, planning and management and thus can offer better services at possibly low costs. Costs will mean not only the resources directly spent on transportation but also secondary impacts on the environment (by exhausts, noise etc.). When discussing an intelligent transportation system, intelligence or an intelligent system is to be defined at first. The intelligence (used in an ITS) can be generally defined as:

"Ability to perceive logical relationships and use one's knowledge to solve problems and respond appropriately (or in an optimal way) to novel situations."

Artificial intelligence in computers is supposed to be a "capability of performing some functions usually associated with human reasoning".

The above mentioned definitions show the common features of an intelligent system, which should accumulate information about the system and its environment, evaluate it in a creative way and choose an optimal way of future behaviour or an optimal control of a system.

Intelligent transportation system should comply with these definitions and accumulate, evaluate an information about the state of the system and use it for an appropriate control of transportation processes. This definition can be used for any process or task in transportation systems. A vehicle should pick up information on a road, vehicle itself and traffic on the road and control its behaviour according to this information to minimise a consumption, to minimise an impact on the environment, to minimise a travel time etc.

The optimisation can be done on a level of a dispatcher control, when a dispatcher can choose a suitable vehicle and its path for a demanded transport. ITS can use a navigation system GPS (Global Positioning System) for a localisation of a vehicle and finding an optimal path to a destination. Information systems on transportation services (free parking places, traffic density on roads, time tables for transit systems etc) serve similarly on a level of a dispatcher control. Optimisation on this level should find an optimal (shortest, fastest, cheapest) path to a destination in a network, optimal routing for a collection or a distribution of goods or passengers, optimal allocation of resources (vehicles, crews) etc.

A strategic decision can include optimal location of transportation and logistic centres or a network design problem, which seeks for an optimal set of connections in a network to serve transportation demands. Practical examples of a location of centres can be location of warehouses, railway or bus stations or postal boxes. The network design can be interpreted also as an optimal choice of roads or railroads to be built or reconstructed, telephone lines or connections among computers in a computer network, which are all strategic and long lasting decisions. The same task can also be defined for some transport services to be introduced such as bus lines, direct freight trains, air lines etc, which are just organisational decisions and can be done or changed with much lower costs.

To summarise the introductory notes: ITS introduces some extra "intelligence" into transportation systems, so that they can provide better services either at a higher quality level or by less operational costs. The way how to achieve these goals is to add a subsystem for collection and evaluation of necessary information, modelling of systems and optimisation methods for a choice of optimal decisions.

There has been no mention about artificial intelligence so far, which is known from computer science. The necessary intelligence can be added to a transportation system as a technical subsystem but can be also represented by a human operator who will show the characteristics of intelligence described in the introduction. Specifically, he will be able to use the available information

\footnotetext{
* Petr Cenek

Department of transportation networks, Faculty of Management Science and Informatics, University of Žilina
} 
about the state of a system in an active and creative way and together with his own knowledge to insure an optimal control of a system.

\subsection{Transportation system}

The transportation system ensures the transportation of different commodities in a transportation network to a final destination in a demanded time and with an appropriate quality (comfort, safety, without a damage etc). Transportation supposes a "mass production", which means a repeated service for many customers or a common transport of many items at a time. The aspect of a mass production can be seen in a sense of:

- transported quantity (many passengers or pieces of goods at a time by one vehicle);

- repetition in time meaning, for instance, repeated transports on a public road shared by many users during a long time period.

A transportation system consists of:

- a fixed subsystem (transportation network or infrastructure), which is composed of nodes and edges of a network,

- a moving subsystem (vehicles and transportation flows),

- a control subsystem.

The quality improvements can be done in nodes or along edges of a network or can be done in a transportation system as a whole. The improved control can be also designed for various transportation modes (as a railway, road, air or water transport, postal and logistics services, combined and container transportation).

\subsection{Systems control}

The improved quality of a transportation system control was mentioned as a main goal of introducing ITS. That is why general principles of the control theory should be respected.

A classical approach is a closed loop control where a control subsystem collects the information about a controlled system, compares this information to defined target values and according to this evaluation decides on the control decisions. A similar approach can be applied for a control of transportation systems, namely a collection of information on a state of the system, evaluation of experiments with a model of a transportation system with various control strategies and choice of the best strategy for future control of the system.

\section{Processes in transportation systems}

As already mentioned, the control tasks can be approached on different levels, which means for individual vehicles, at nodes and edges of a network or for a transportation system as a whole. These different control problems will be discussed in further chap- ters and at least some examples of possible quality improvements by an improved control in ITS will be shown.

\subsection{Vehicles}

Control of individual vehicles is a problem of a control theory; even if there is an important difference to other technical systems in a fact that a human operator (a driver) usually operates vehicles. A human operator can use his intelligence to react in an active and creative way on various traffic situations, but he is also the least reliable element of the control subsystem. There are several topics to be approached when improving the control on a vehicle level:

- a man - machine system, driver's reactions on traffic situations and driver's psychology,

- introduction of "artificial intelligence" into a vehicle control subsystem using technical tools such as sensors for an obstacle and/or other vehicles detection, evaluation systems which recognise the traffic situation on a road and recommend a suitable way of driving or possibly give warning signals to prevent dangerous states,

- enhancement of vehicles "intelligence" using communication tools which enable an automatic communication among vehicles; such a communication system provides timely information for following vehicles on a future dangerous states recognised by a leading vehicle or on a critical behaviour of the leading vehicle (for instance, emergency breaking in front of an obstacle on a road); the following vehicles can then react in advance and so they can avoid collisions and in less critical situations may solve the situation at smaller costs (less intensive breaking for instance).

- a global positioning system GPS with other communication systems can similarly improve the quality of a vehicle control; vehicles can be informed about their own position and receive data necessary for their navigation (for finding a path to a destination), but may be also informed about a position of other vehicles and obstacles at their proximity even if such obstacles are invisible for a driver (behind a curve or in conditions of a limited visibility); again it is possible to avoid critical situations or to solve the critical situation at smaller costs,

- systems of a fully automatic vehicle control are the highest level of a vehicle control; such systems will be able to follow automatically the road to a desired destination, choose the optimal path, recognise and respect traffic on a road and prevent from any collisions with other vehicles moving in the same lane (longitudinal collisions) or with vehicles in other lanes (lateral collisions). Automatic control can be accomplished using many different technical devices such as various sensors, balises built in a road and read by sensors in a vehicle or precise satellite navigation systems GPS.

All the mentioned approaches are already technically solvable and automatic guided vehicles are already used on private roads within some industrial plants, amusement parks etc. A general acceptance of such systems is prevented by high costs, insufficient safety, necessity of specialised roads equipped with technical tools 
(balises or inductive loops) and low speed of automatically guided vehicles (due to safety in public traffic). Possible collisions with other vehicles and pedestrians in traffic on public roads represent another type of obstacles for an allowance of such systems in a general use.

\subsection{Processes on edges of a network}

The edges of a network serve for motion of vehicles and transported commodities. The transportation of commodities along an edge is a basic process in all transportation systems and represents the main goal of a transportation activity - the displacement of passengers or other commodities in a transportation system. There are many vehicles running simultaneously on a road and so mutual conflicts have to be avoided. The vehicles must respect safety distances among them, they have to brake and accelerate according to a current traffic situation or they have to overpass slower vehicles. The mutual interaction of vehicles is examined in a traffic flow theory.

An improved quality of ITS control should offer better traffic control on a road, which would prevent any collisions and ensure a fluent motion of vehicles with a minimum fuel consumption and minimum negative impacts on the environment.

The priority goal should be in many cases to enhance a road capacity (the maximum number of vehicles which can go along a road per a time unit). Another important goal is improved safety of traffic. The goals can be achieved by various technical improvements:

- better dynamics of a vehicle (more effective brakes and higher performance of an engine) allows to decrease a necessary safety distance between vehicles and to increase a cruising speed and thus a road capacity can be enhanced,

- the same result can be achieved by reducing a reaction time of a vehicle control subsystem; the reaction time can be decreased by various technical tools for instance by sensors which measure the distance from the leading vehicle and an alert system for a driver or a direct automatic emergency braking; another system may warn a driver about an approaching obstacle on the road.

- the capacity of a railroad can be increased similarly by introducing new interlocking systems, which allow to decrease a follow up distance among the trains.

The problems of a traffic control are quite different in road transport and railway (or rail bound) transport. There is a vast number of possible situations in a road transport which can lead to a conflict with a leading or following vehicle, with a vehicle in an opposite direction or in other road lanes, because vehicles can use any part of the road (any lane). The situation is different in a railway transport, where trains can use only one rail track (or a section of a track) allocated to the train and so only longitudinal conflicts among trains are possible. A variety of conflict situations in a railway transport is thus limited. On the other hand, trains are bound to one lane (one track) only, cannot overpass other trains on a track and they have in general also worse dynamic characteristics. That is why the capacity of railroads is fairly low, especially when trains of different categories move on the track.

\subsection{Processes in nodes of a network}

Control of processes in a node of a network is much more diversified than on an edge. The node of a network can represent a railway station, a crossing of roads, a village or a town. There are many roads in a node where vehicles can stand or move along. A "mass production" or displacement of many items at a time was described as one of characteristics of a transportation system. This brings new problems of accumulation and sorting of items to be transported. The batches of items can be created by:

- accumulation of items which enter the node from outside world (passengers come to a station or postal parcels are brought into a post office)

- sorting from incoming vehicles or trains, which brought the transported items into a node and such items have to be sorted according to their destination address.

The node of a network usually represents a transportation network by itself (on a more detailed level of a model). Such a network is created by streets in a town or by trackage in a railway station. There are typically many vehicles, which must be parked on roads or move along them. All the vehicles compete for a position on a road, which is to be shared among the vehicles. The individual vehicles thus represent parallel processes, which have to be co-ordinated in a system. There are no grave problems in a real traffic, where human operators drive vehicles. Drivers know and respect traffic regulations and can creatively solve some exceptions, which may arise in a real traffic.

To introduce intelligence in such systems by technical means is quite a difficult task. An example of such a problem may be a simulation model of traffic in a node, where all decisions have to be modelled in a simulation program. The lack of a human intelligence and its creativity in solving exceptional situations can result in a deadlock in a simulation program. The system enters a situation, which cannot be solved by regular means (no process can continue and be finished) and so vehicles are stacked in their current positions and the simulation run must be stopped. The coordination of parallel processes is one of the most demanding tasks in a control of transportation processes.

\subsection{Processes in a transportation system}

Processes on a level of the whole transportation system should mainly serve to move the commodities among the nodes of a network. The optimisation will concentrate on the optimisation of paths in the network, or problems of space and time planning. The decisions can be done on several levels:

- strategic level decides on basic problems of building of a transportation system such as location of nodes and centres in a network, building of new edges (roads, railway tracks or waterways). The optimisation problems are typically of a combinatorial 
nature and are very difficult to solve. On the other hand, the importance of such decisions is long-lasting and that is why it is possible to spend quite a long computation time to find the best alternative,

- tactical level can also decide on location of centres and building some relations to serve the transportation needs in a network; this time the interpretation of the problem is not building a physical infrastructure but rather taking organisational decisions (which trains and/or bus lines should operate, time tables, location of bus stops etc)

- operational level organises a work of individual vehicles, finds shortest paths and optimal paths for distribution and collection of transported items.

Planning and management on the level of ITS should utilise various optimisation methods to find optimal solutions on each control level.

\section{Implementation of ITS}

Intelligent transportation systems are usually implemented according to a transportation mode and to a service, which is offered to customers. This approach is typical for practical needs when an existing problem should be solved in a possibly fast and efficient way. This can lead to a redundant solving of similar problems, to a variety of different solutions and to their possible incompatibility.

The research in ITS can be organised in the opposite way, too, which means it should concentrate on general formulation of problems and common methods of control in ITS. The developed general tools can be then used in practical applications. A general acceptation of solutions, possibility of mutual communication and a unification of used tools and control methods is a desired characteristic of ITS. In this way a standard data on transportation networks will be used, a standard user environment can be offered to a user and at least basic optimisation tasks can be solved using standard well tested optimisation methods. This approach of a problem generalisation, solution of general problems and application of results is typical for a research at universities and that is why these general problems will be discussed in more detail.

\subsection{Model of a transportation infrastructure}

Transportation infrastructure is characterised by linear objects (roads, railroads, and waterways) and that is why linear co-ordinates are to be used. Therefore, locations in a transportation system will not be defined by Cartesian co-ordinates but will be addressed by a reference to an element (edge) of a network and by its longitudinal co-ordinate (distance from a beginning of the edge). The objects not lying directly on the network (on a road) can be addressed in a similar way. In this case, two co-ordinates will be used besides the reference on the network element. The first coordinate will again describe the distance (longitudinal co-ordinate) and the second co-ordinate will describe the distance from the nearest point in the network (lateral co-ordinate). Linear co-ordinate system is currently being used in many transportation systems and it offers a simple, unambiguous and generally valid identification of all objects in a transportation system.

Data on a network are typically vast files and their input is a costly process. Therefore, it is reasonable to input the data only once and then to share them by many users who can use various computers and operating systems. A commercial database provides an access to data for many users using the same operating system. Another tool such as XML document should be used to share the data among many computers with different operating systems.

Another important part of an infrastructure model is its graphical visualisation. A graphical interface is the best tool to provide a quick and understandable information to a user. Graphical output is suitable also for presentation of a transportation network, position of vehicles and their motion in a network.

\subsection{Model of a vehicle and traffic flow}

Vehicles are dynamical systems, states of which (position, speed, acceleration) are changing in time. A motion of several vehicles along the edge of a network must respect mutual interactions and possible conflicts on a road (a necessity of acceleration or braking, surpassing etc). Concurrent motion of several vehicles on a road is known as traffic flow. Some optimisation problems do not deal with a detailed model of a dynamical behaviour of individual vehicles or of a transportation flow as a whole and use only a static value of a flow intensity (or number of vehicles passing a point in a network during a unit of time). The flow intensity can thus replace traffic flow model for a certain class of optimisation problems.

Vehicle models are different for road and railway transports from a point of view of kinematics, dynamics and mutual interactions as well. The trajectory of a vehicle movement is unambiguously defined by a rail track and that is why only dynamics of a vehicle movement in one co-ordinate is controllable (along a rail track forwards or backwards). Similarly, kinematics of the movement is quite simple, as the front wheels and rear wheels must follow the geometry of a railroad. The co-ordination of several vehicles on a track is limited to an allocation of a track (or a part of it) to only one vehicle (train) because vehicles cannot pass each other.

Vehicle motion in a road transport (as well as in water or air transport) is not limited to one co-ordinate, but generally at least two co-ordinates can be controlled (the trajectory is not defined by a road but can be controlled by a driver). Kinematics is much more complicated because only the front vheels follow the chosen path while the rear wheels are pulled freely and follow the preceding positions of the front wheels. Similarly, speed of a train motion is defined by a railroad construction, while the speed must comply with a chosen trajectory in a road transport. The speed will thus be limited by a radius of a trajectory curve, by a traffic situation etc. 
A different situation is also at co-ordination of several vehicles, when drivers and vehicles are fairly free in a choice of speed and trajectory (lane). The traffic must respect also vehicles in the opposite direction, which is in a railroad transport unthinkable as they are strictly separated.

Models of vehicles can be very different from each other and must reflect all the characteristic of a chosen transportation mode.

\subsection{Information systems - collection, processing and storage of information}

Control in a closed loop supposes an availability of an information about a state of a controlled system and a comparison of these data with a demanded goal, which allows generating appropriate control actions. The same approach can be used in a control of transportation systems or processes in transportation systems. A characteristic of a transportation system is that it is geographically widespread, which means that elements of the system are located in distant places and the information must be collected over information networks. Transportation systems differ from other technical systems also in an expected response time, which is usually quite long with transportation systems, typically in order of minutes (with an exception of direct driver response).

A perfect and timely information is crucial for a control of transportation processes. For instance, if there is a direct communication with vehicles and fast (on time) information on transportation demands, the vehicle fleet can be managed in much more effective way than when the control can be executed only in depots. Similarly the planes can be better exploited, or the trains can have only necessary number of wagons according to the estimated demand.

A use of telematic systems is an efficient way of collecting and processing information in distributed systems. Telematic systems combine telecommunication services for data transmissions with processing power of computers or computer networks. The effectiveness of such a control was proved by practical experience, when many transportation companies gained a significant competitive advantage by using those systems. That is why they may grow up, provide better quality of service at lower operational costs.

\subsection{Control and optimisation methods}

The last part of an efficient control subsystem is a suitable optimisation method. The input data and model of a transportation system provide a platform for experiments and search of optimal control decisions. There are several basic approaches how to find an optimal (or sub optimal) decision:

- exact methods of operations research or graph theory, which use a mathematical formulation of a problem and an algorithm will find an optimal solution by a strictly defined and proved process,
- heuristic methods, which use the same mathematical formulation of a problem, but the algorithms are based on a human experience with solving similar problems and give a possibly good sub- optimal solution; heuristic methods are used preferably for complex combinatorial problems, for which an optimal solution by exact methods would be unrealistic,

- simulation methods, which are used for modelling and analysis of stochastic systems, with random input values or changes in the system characteristics,

- interactive methods, which profit from an operator's experience with solving similar problems and his ability of fast space orientation in the graphical presentation of a problem; interaction of an operator with a computer can be also used to respect some additional constraints, which are hardly to be formulated mathematically,

- artificial intelligence uses expert knowledge of a system and proposes suitable decisions; the artificial intelligence is best suited to solve problems, where the solution is to be chosen from a number of variants.

\section{Conclusions}

The University of Žilina has been interested in optimisation of transportation processes for a long time. Developments and construction of ITS can profit from this experience and utilise the results of research works. Many control methods discussed in the paper are theoretically known, some of them are already implemented and directly used in practice. Some principles and methods are just suggestions for further research work. These problems encompass:

- Integration of available models and control tools into a unified environment and communication with other subsystems;

- Development of new elements of a control subsystem, which may be directed to

- Design of a general model of a transportation infrastructure with graphical output [5], choice of relevant parameters for elements of a network, design of a standard database for transportation networks and design of a general template and procedures for storage, reading and transfer of XML documents describing a transportation network;

- Design of a general model of a vehicle including dynamics, visualisation and animation routines [2];

- Development of new and modifications of known optimisation methods suitable for a general model of a transportation network [1], [3], [4];

- Research concerning new control problems such as control of parallel processes in transportation systems, control using time tables and construction of time tables and control under incomplete information and/or influence of a quality and availability of information on a quality of transportation process.

- Practical application of developed control tools in various transportation modes and for different transportation processes. 


\section{KOMNIIKOCle}

\section{References}

[1] CENEK P., KLIMA V., JANÁČEK J.: Optimisation of Transport and Telecommunication systems (Optimalizace dopravních a spojových systémů). EDIS-ZU, Žilina 1994

[2] CENEK P.: Vehicle Kinematics and Microsimulation Models. Journal of Information, Control and Management Systems, Vol. 1, No. 1, FRI-ŽU, Žilina 2003

[3] JANÁČEK J.: Mathematical Programming (Matematické programování). EDIS-ŽU, Žilina 1999

[4] JANÁČEK J.: Optimisation of Transport Systems (Optimalizace dopravních systémů). EDIS-ŽU, Žilina 1999

[5] JÁNOŠÍKOVÁ L., SADLOŇ L., CENEK J.: Model of Intelligent Transportation Infrastructure. Journal of Information, Control and Management Systems, Vol. 1, No. 1, FRI-ŽU, Žilina 2003. 